\title{
Non-Equilibrium 2PI Potential and Its Possible Application to Evaluation of Bulk Viscosity*
}

\author{
Yu. B. Ivanov ${ }^{1,0}$ 团 and D. N. Voskresensky ${ }^{2}$, 团 \\ ${ }^{1}$ NRC "Kurchatov Institute", Kurchatov sq. 1, Moscow 123182, Russia \\ ${ }^{2}$ National Research Nuclear University "MEPhI", Kashirskoe sh. 31, Moscow 115409, Russia
}

\begin{abstract}
Within non-equilibrium Green's function technique on the real-time contour and the two-particleirreducible (2PI) $\Phi$-functional method, a non-equilibrium potential is introduced. It naturally generalizes the conventional thermodynamic potential with which it coincides in thermal equilibrium. Variations of the non-equilibrium potential over respective parameters result in the same quantities as those of the thermodynamic potential but in arbitrary non-equilibrium. In particular, for slightly non-equilibrium inhomogeneous configurations a variation of the non-equilibrium potential over volume is associated with the trace of the non-equilibrium stress tensor. The latter is related to the bulk viscosity. This provides a novel way for evaluation of the bulk viscosity.
\end{abstract}

\section{INTRODUCTION}

Non-equilibrium Green's function technique, developed by Schwinger, Kadanoff, Baym and Keldysh [1 [5], is the appropriate concept to study the space-time evolution of many-particle quantum systems. This formalism finds now applications in various fields, such as quantum chromo-dynamics [6], nuclear physics [7] 19$]$, astrophysics [10, 20, 21], cosmology [22, 23], spin systems [24], lasers [25], plasma physics [26, 27], physics of liquid ${ }^{3} \mathrm{He}$ [28], critical phenomena, quenched random systems and disordered systems [29], normal metals and super-conductors [20, 30, 31], semiconductors [32], tunneling and secondary emission [33], ultra-cold gases [34], etc. This list is certainly not complete.

With the aim to come to a tractable dynamical scheme one compulsory performs partial re-summations. In contrast to perturbation theory, however, doing re-summations one frequently encounters a complication, that the resulting equations of motion may no longer comply with the conservation laws, e.g., of charge, energy and momentum. This problem was considered in [35] in terms of equilibrium Green's function formalism. Any approximation, in order to be conserving, must be so-called $\Phi$-derivable, see also [36, 37]. Any $\Phi$-derivable approximation provides thermodynamically consistent results. A common generating functional depending on auxiliary two-particle irreducible (2PI) $\Phi$ functional was then constructed on equilibrium [38] and non-equilibrium [12] Schwinger-Keldysh contours, being determined in terms of full, i.e. re-summed, classical fields and Green's functions coupled by free vertices. The presented scheme of constructing self-consistent approximations provides a suitable basis for the derivation of generalized kinetic description beyond the limitations of the quasi-particle approximation [13 18]. Such generalized transport schemes respect the quantum nature of the particles and, that is of particular importance, take into account their finite mass-widths. Relativistic effects first introduced in quantum kinetics by Belyaev and Budker [39] are easily incorporated in the mentioned general scheme.

Close to equilibrium, at time intervals much larger than the time scale of changes of kinetic quantities the generalized kinetic description can be replaced by a more economical hydrodynamical description. The fluid-dynamical approach is fairly efficient for description of heavy-ion collisions in a broad incident energy range from SIS to LHC energies. Interest in the transport coefficients within the hydrodynamics has been raised by large values of elliptic flow at RHIC energies [40], though their study started long before [41, 42]. The observed elliptic flow indicated that the created quark-gluon plasma behaves like a fluid with a low (but non-zero) value of the shear viscosity [43]. The bulk viscosity can also essentially affect the dynamics of heavy-ion collisions, supernovas in astrophysics and acceleration of the Universe in cosmology [44]. As it was shown in [45], large value of the bulk viscosity can result in instability at the freeze-out stage. A contribution to the pressure related to the bulk viscosity affects the equation of state, making it softer for expanding systems and stiffer for contracting ones 19]. The stability of neutron stars to the growth of $r$-modes is provided only by large values of the bulk viscosity of the matter [46]. Transport coefficients also govern the dynamics of the first-order phase transitions [4].

\footnotetext{
* Dedicated to S.T. Belyaev on the occasion of his 90th birthday. †Y.Ivanov@gsi.de

†D.Voskresensky@gsi.de
} 
Evaluation of transport coefficients of strongly interacting matter is usually done within the quasiparticle approximation [48], although the width effects can be large at least for some species. Studies of transport coefficients for resonances have just recently started [19, 49]. Moreover, all above derivations were performed within simplified approximation, i.e. the relaxation time approximation. A rigorous way to evaluate transport coefficients is based on Kubo formulas [50]. Calculations of such kind are highly complicated [51, 52]. In [53] the problem was discussed in the framework of the $2 \mathrm{PI}$ method. The attention was primarily focused on the shear viscosity. In the present paper we mainly address the bulk viscosity as one of the possible applications of the introduced non-equilibrium 2PI potential. The case of the bulk viscosity is extra complicated. In addition to the conventional kinetic contribution related to single-particle dynamics, the bulk viscosity contains an important contribution coming from fluctuations of soft collective modes [54, 55]. The latter contribution appreciably increases in the vicinity of the phase transition [56]. Therefore, a reliable scheme for calculating the bulk viscosity should properly incorporate both these contributions.

The aim of the present paper is introduction of a non-equilibrium potential that generalizes the conventional thermodynamic potential beyond the scope of equilibrium. We describe one of the possible applications of this new potential, i.e. its application to evaluation of the bulk viscosity proceeding from $\Phi$-derivable approximations within non-equilibrium Green's function technique. In sect. [I] we briefly formulate concepts developed in [12]. In sect. III we derive a non-equilibrium potential, based on the generating functional, and show that the non-equilibrium potential transforms into the conventional thermodynamic potential in thermal equilibrium. Then in sect. [IV] we discuss relations between the non-equilibrium potential, the pressure, the stress tensor and the bulk viscosity. Some helpful relations are deferred to the Appendix $\mathrm{A}$

\section{PREREQUISITES}

In this section we briefly formulate concepts developed in [12] and introduce necessary notations.

\section{A. Noether Energy-Momentum Tensor and Current}

For notational convenience we consider a system of relativistic scalar bosons, specified by free Klein-Gordon Lagrangians

$$
\widehat{\mathcal{L}}^{0}= \begin{cases}\frac{1}{2}\left(\partial_{\mu} \widehat{\phi} \cdot \partial^{\mu} \widehat{\phi}-m^{2}(\widehat{\phi})^{2}\right) & \text { for neutral bosons } \\ \left(\partial_{\mu} \widehat{\phi}^{\dagger} \cdot \partial^{\mu} \widehat{\phi}-m^{2} \widehat{\phi}^{\dagger} \widehat{\phi}\right) & \text { for charged bosons }\end{cases}
$$

where $\widehat{\phi}(x)$ and $\widehat{\phi}^{\dagger}(x)$ are bosonic field operators. All considerations are straight forwardly adapted to multicomponent systems of different flavors, to fields with intrinsic degrees of freedom, to non-scalar fields, such as vector boson or Dirac fermion fields or also to non-relativistic many-body pictures. The interaction Lagrangians $\widehat{\mathcal{L}}^{\text {int }}\{\widehat{\phi}\}$ (for neutral bosons) and the charge symmetric $\widehat{\mathcal{L}}^{\text {int }}\left\{\widehat{\phi}, \widehat{\phi}^{\dagger}\right\}$ for charged bosons are assumed to be local, i.e. without derivative coupling. Generalization to the derivative coupling interaction can be performed following the line of [16].

The variational principle of stationary action leads to the Euler-Lagrange equations of motion for the field operators

$$
S_{x} \widehat{\phi}(x)=-\widehat{J}(x)=-\frac{\partial \widehat{\mathcal{L}}^{\text {int }}}{\partial \widehat{\phi}^{\dagger}}, \quad \text { where } \quad S_{x}=-\partial_{\mu} \partial^{\mu}-m^{2}
$$

and similarly for the corresponding adjoint equation. Thereby the $\widehat{J}(x)$ operator is a local source current of the field $\widehat{\phi}$, while $S_{x}$ is the differential operator of the free evolution with the free propagator $\Delta^{0}(y, x)$, as resolvent.

The canonical form [57] of the energy-momentum tensor operator reads

$$
\widehat{\Theta}_{(\text {canon. })}^{\mu \nu}=\kappa\left(\frac{\partial \widehat{\mathcal{L}}}{\partial\left(\partial_{\mu} \widehat{\phi}\right)} \partial^{\nu} \widehat{\phi}+\frac{\partial \widehat{\mathcal{L}}}{\partial\left(\partial_{\mu} \widehat{\phi}^{\dagger}\right)}\left(\partial^{\nu} \widehat{\phi}^{\dagger}\right)\right)-g^{\mu \nu} \widehat{\mathcal{L}},
$$

which is conserved, i.e. $\partial_{\mu} \widehat{\Theta}_{\text {(canon.) }}^{\mu \nu}=0$. Here and below $\kappa=1 / 2$ for neutral bosons and 1 for charged bosons, $g^{\mu \nu}=$ $\operatorname{diag}(1,-1,-1,-1)$. The canonical energy-momentum tensor is non-symmetric. Alternatively, using equations of 
motion (2.2) one can derive symmetric expression for the conserved energy momentum tensor. Introducing convenient notation $\widehat{p}_{x}^{\mu}=\mathrm{i} \partial_{x}^{\mu}$ we rewrite the symmetric energy-momentum tensor as

$$
\begin{aligned}
\widehat{\Theta}^{\mu \nu}(x) & =\frac{1}{4} \kappa\left[\left(\left(\widehat{p}_{x}^{\mu}\right)^{*}+\widehat{p}_{y}^{\mu}\right)\left(\left(\widehat{p}_{x}^{\nu}\right)^{*}+\widehat{p}_{y}^{\nu}\right)\left[\widehat{\phi}^{\dagger}(x), \widehat{\phi}(y)\right]\right]_{x=y} \\
& +g^{\mu \nu}\left(\widehat{\mathcal{E}}^{\text {int }}(x)-\widehat{\mathcal{E}}^{\text {pot }}(x)\right) .
\end{aligned}
$$

Here $[\ldots, \ldots]$ denotes commutator and we introduced operators of the interaction-energy density $\widehat{\mathcal{E}}^{\text {int }}$ and the potential-energy density $\widehat{\mathcal{E}}^{\text {pot }}$ :

$$
\begin{gathered}
\widehat{\mathcal{E}}^{\text {int }}(x)=-\widehat{\mathcal{L}}^{\text {int }}(x) \\
\widehat{\mathcal{E}}^{\text {pot }}(x)=-\frac{1}{2} \kappa\left(\widehat{J}^{\dagger}(x) \widehat{\phi}(x)+\widehat{J}(x) \widehat{\phi}^{\dagger}(x)\right) .
\end{gathered}
$$

It is easy to show that $\partial_{\mu}\left(\widehat{\Theta}^{\mu \nu}-\widehat{\Theta}_{(\text {canon. })}^{\mu \nu}\right)=0$. This implies that $\widehat{\Theta}^{\mu \nu}$ and $\widehat{\Theta}_{(\text {canon.) }}^{\mu \nu}$ are equivalent.

For specific interaction Lagrangians, e.g., with a certain number $\gamma$ of operators attached to each vertex, eq. (2.6) together with definition (2.2) for the current $\widehat{J}$ allow to obtain a simple relation between $\widehat{\mathcal{E}}^{\text {pot }}$ and $\widehat{\mathcal{E}}^{\text {int }}$,

$$
\widehat{\mathcal{E}}^{\text {int }}(x)=\frac{2}{\gamma} \widehat{\mathcal{E}}^{\text {pot }}(x) \text {. }
$$

For $\phi^{4}$-theory $\gamma=4$.

If the Lagrangian is invariant under some global transformation of complex fields (with the charge $e$ ), e.g.,

$$
\widehat{\phi}(x) \Rightarrow e^{-\mathrm{i} e \Lambda} \widehat{\phi}(x) ; \quad \widehat{\phi}^{\dagger}(x) \Rightarrow e^{\mathrm{i} e \Lambda} \widehat{\phi}^{\dagger}(x)
$$

there exists Noether current defined as [57],

$$
\widehat{j}_{(\text {Noether })}^{\mu}(x)=e \frac{1}{2}\left[\left(\left(\widehat{p}_{x}^{\mu}\right)^{*}+\widehat{p}_{y}^{\mu}\right)\left[\widehat{\phi}^{\dagger}(x), \widehat{\phi}(y)\right]_{\mp}\right]_{x=y} .
$$

which is conserved, i.e. $\partial_{\mu} \hat{j}_{(\text {Noether) }}^{\mu}=0$. This current naturally vanishes for neutral fields.

\section{B. Real-Time Contour}

In the non-equilibrium case, one assumes that the system has been prepared at some initial time $t_{0}$ in terms of its density operator $\widehat{\rho}_{0}=\sum_{a} P_{a}|a\rangle\langle a|$ at that time, where $|a\rangle$ form a complete set of eigenstates of $\widehat{\rho}_{0}$.

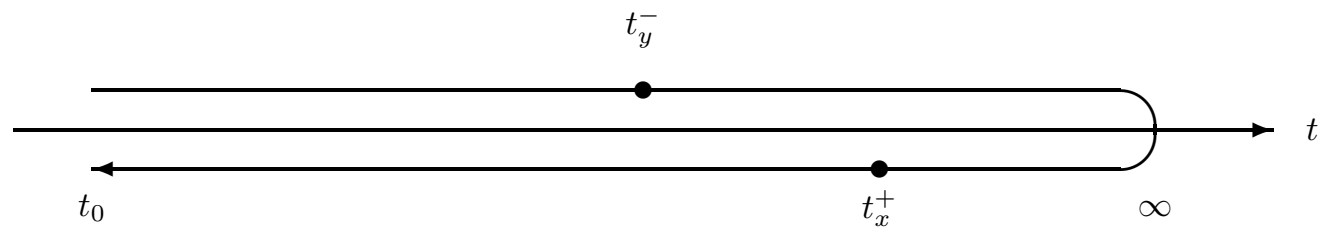

Figure: Closed real-time contour with two external points $x, y$ on the contour.

The non-equilibrium theory can be formulated on a closed real-time contour (see figure), where the time runs from $t_{0}$ to $\infty$ along time-ordered branch and back to $t_{0}$ along anti-time-ordered branch. Contour-ordered multi-point functions are defined as expectation values of contour-ordered products of operators,

$$
\left\langle\mathcal{T}_{\mathcal{C}} \widehat{A}\left(x_{1}\right) \widehat{B}\left(x_{2}\right) \ldots\right\rangle=\left\langle\mathcal{T}_{\mathcal{C}} \widehat{A}_{\mathrm{I}}\left(x_{1}\right) \widehat{B}_{\mathrm{I}}\left(x_{2}\right) \ldots \exp \left\{\mathrm{i} \int_{\mathcal{C}} \widehat{\mathcal{L}}_{\mathrm{I}}^{\mathrm{int}} \mathrm{d} x\right\}\right\rangle
$$


where $\mathcal{T}_{\mathcal{C}}$ orders the operators according to a time parameter running along the contour $\mathcal{C}$. The l.h.s. is written in the Heisenberg representation, the r.h.s., in the interaction picture (subscript "I").

In certain calculations, e.g., in those that apply Fourier and Wigner transformations, it is necessary to decompose the full contour into its two branches - the time-ordered and anti-time-ordered branches. One then has to distinguish between the physical space-time coordinates $x, \ldots$ and the corresponding contour coordinates $x^{\mathcal{C}}$, which for a given $x$ take two values $x^{-}=\left(x_{\mu}^{-}\right)$and $x^{+}=\left(x_{\mu}^{+}\right)(\mu \in\{0,1,2,3\})$ on the time ordered and anti-time ordered branches, respectively (see figure):

$$
\int_{\mathcal{C}} \mathrm{d} x^{\mathcal{C}} \ldots=\int_{t_{0}}^{\infty} \mathrm{d} x^{-} \ldots+\int_{\infty}^{t_{0}} \mathrm{~d} x^{+} \ldots=\int_{t_{0}}^{\infty} \mathrm{d} x^{-} \ldots-\int_{t_{0}}^{\infty} \mathrm{d} x^{+} \ldots
$$

where only the time limits are explicitly given. One-point functions have the same value on both sides on the contour. The corresponding properties of two-point functions and their equilibrium relations are summarized in Appendix A. For any two-point function $F$ the contour values are defined as $F^{i j}(x, y):=F\left(x^{i}, y^{j}\right), \quad i, j \in\{-,+\}$ on the different branches of the contour, cf. A.1 . Due to the change of operator ordering genuine multi-point functions are discontinuous in general, when two contour coordinates become identical.

Boson fields may take non-vanishing expectation values of the field operators $\phi(x)=\langle\hat{\phi}\rangle$, called mean fields, or classical fields. The corresponding equations of motion are provided by the ensemble average of the operator equations of motion (2.2)

$$
S_{x} \phi(x)=-J(x), \quad \text { or } \quad \phi(x)=\phi^{0}(x)-\int_{\mathcal{C}} \mathrm{d} y \Delta^{0}(x, y) J(y) .
$$

Here $J(x)=\langle\mathrm{J}(x)\rangle$, while $\phi^{0}(x)=\left\langle\widehat{\phi}_{\mathrm{I}}(x)\right\rangle$ is the freely evolving classical field, which starts from $\phi\left(t_{0}, \boldsymbol{x}\right)$ at time $t_{0}$. The free contour Green's function

$$
\begin{aligned}
\mathrm{i} \Delta^{0}(x, y & =\left\langle\mathcal{T}_{\mathcal{C}} \widehat{\phi}_{\mathrm{I}}(x) \widehat{\phi}_{\mathrm{I}}^{\dagger}(y)\right\rangle-\phi^{0}(x)\left(\phi^{0}(y)\right)^{*} \quad \text { is resolvent of } \\
S_{x} \Delta^{0}(x, y) & =\delta_{\mathcal{C}}(x, y), \quad \text { with } \quad \delta_{\mathcal{C}}\left(x^{i}, y^{j}\right)=\sigma^{i j} \delta^{4}(x-y)=\left(\begin{array}{cc}
1 & 0 \\
0 & -1
\end{array}\right) \delta^{4}(x-y) .
\end{aligned}
$$

One can easily verify the equivalence of the contour form (2.12) with the standard retarded classical field equation and the fact that $J(x)$ and $\phi(x)$ are one-point functions, which have identical values on both sides of the contour.

Subtracting the classical fields via $\widehat{\phi}=\phi+\widehat{\varphi}$, the full propagator in terms of quantum-fluctuating parts $\widehat{\varphi}$ of the fields is defined as

$$
\mathrm{i} \Delta(x, y)=\left\langle\mathcal{T}_{\mathcal{C}} \widehat{\varphi}(x) \widehat{\varphi}^{\dagger}(y)\right\rangle=\left\langle\mathcal{T}_{\mathcal{C}} \widehat{\phi}(x) \widehat{\phi}^{\dagger}(y)\right\rangle-\phi(x) \phi^{*}(y)=\left\langle\mathcal{T}_{\mathcal{C}} \widehat{\phi}(x) \widehat{\phi}^{\dagger}(y)\right\rangle_{c} .
$$

Index "c" indicates that uncorrelated parts are subtracted. In terms of diagrams it implies, that the corresponding expectation values are given by sums of connected diagrams.

Averaging the operator equations of motion (2.2) multiplied by $\widehat{\phi}^{\dagger}(x)$ and subtracting classical field parts one obtains the equation of motion for the propagators,

$$
S_{x} \Delta(x, y)=\delta_{\mathcal{C}}(x, y)+\int_{\mathcal{C}} \mathrm{d} z \Pi(x, z) \Delta(z, y)
$$

and similarly for the corresponding adjoint equation. $\Pi$ denotes the proper self-energy of the particle. Since we have separated the full propagator in (2.14), -i ll has to be one-particle irreducible (label 1PI), i.e. the corresponding diagram cannot be split into two pieces, which separate $x$ from $z$ by cutting a single propagator line. Obviously, $\Pi$ can have singular ( $\delta$-functional) one-point parts and genuine two-point parts (the latter are given by all connected 1PI diagrams of the current-current correlator),

$$
-\mathrm{i} \Pi(x, z)=\left\langle\mathcal{T}_{\mathcal{C}} \frac{\partial^{2} \mathrm{i} \widehat{\mathcal{L}}^{\mathrm{int}}(x)}{\partial \widehat{\phi} \partial \widehat{\phi}^{\dagger}}\right\rangle_{c} \delta_{\mathcal{C}}(x, y)-\left\langle\mathcal{T}_{\mathcal{C} \mathrm{J}}(x) \mathrm{J}^{\dagger}(y)\right\rangle_{1 \mathrm{PI}},
$$

in the Heisenberg picture.

In diagrams free and full classical fields are represented by "pins" with cross and "o-cross" as heads, cf. (2.17), while free and full propagators are given by thin and thick long lines, respectively. Thereby, complex fields carry a 
sense, the arrow always pointing towards the $\widehat{\phi}$ in the contour ordered expressions. In diagrammatic representation, the classical field equations (2.12) and Dyson's equation (2.15) are then given by

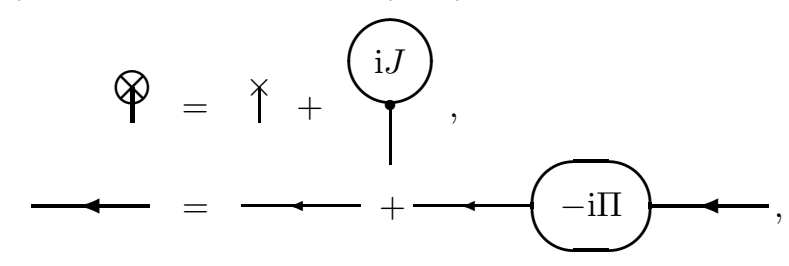

with the one- and two-point functions $\mathrm{i} J(x)$ and $-\mathrm{i} \Pi(x, y)$, as driving terms.

\section{Generating Functional $\Gamma$ and $\Phi$}

In [12] we have constructed the generating real-time $\Gamma$-functional in the form

$$
\begin{aligned}
& \Gamma\left\{\phi, \phi^{*}, \Delta, \lambda\right\}=\Gamma^{0}+\int_{\mathcal{C}} \mathrm{d} x \mathcal{L}^{0}\left\{\phi, \partial_{\mu} \phi\right\} \\
& +\mathrm{i} \kappa\left[\ln \left(1-\odot \Delta^{0} \odot \Pi\right)+\odot \Delta \odot \Pi\right]+\Phi\left\{\phi, \phi^{*}, \Delta, \lambda\right\},
\end{aligned}
$$

defining the auxiliary functional $\Phi\left\{\phi, \phi^{*}, \Delta, \lambda\right\}$. To construct this functional we introduced a space-time dependent interaction scale $\lambda(x)$ into the variational concept, which scales interaction vertices, i.e.

$$
\widehat{\mathcal{L}}_{\lambda}^{\text {int }}=\lambda(x) \widehat{\mathcal{L}}^{\text {int }}\left\{\widehat{\phi}^{\dagger}(x), \widehat{\phi}(x)\right\}, \quad \mathcal{E}_{\lambda}^{\text {int }}(x)=-\left\langle\widehat{\mathcal{L}}_{\lambda}^{\text {int }}(x)\right\rangle .
$$

The $\Gamma^{0}$ and $\mathcal{L}^{0}$ parts, where $\mathcal{L}^{0}$ is the free classical Lagrangian function, represent the non-interacting parts of $\Gamma$. Thereby, the $\Gamma^{0}$ term solely depends on the unperturbed propagator $\Delta^{0}$ and hence is treated as a constant with respect to functional variations of $\Gamma$. The $\ln (\ldots)$ is understood in the functional sense, i.e. by a series of $n$-folded contour convolutions, denoted by the $\odot$-symbol, formally resulting from the Taylor expansion of the $\ln (1+x)$ at $x=0$. The ln-term accounts for the change of $\Gamma$ due to the self-energies of the particles. These first three terms account for the one-body components in the $\Gamma$. The remaining $\odot \Delta \odot \Pi$ and $\Phi$ terms correct for the true interaction energy part of the partition sum.

Functional variation of $\Gamma\left\{\phi, \phi^{*}, \Delta, \lambda\right\}$ in the form of eq. (2.19) leads to

$$
\begin{aligned}
\delta \Gamma\left\{\phi, \phi^{*}, \Delta, \lambda\right\}=\kappa\left\{\int_{\mathcal{C}} \mathrm{d} x[\delta \phi\right. & \left.(x)\left(S_{x}\right)^{*} \phi^{*}(x)+\delta \phi^{*}(x) S_{x} \phi(x)\right] \\
& -\mathrm{i}\left(\odot \frac{1}{1-\odot \Delta^{0} \odot \Pi} \odot \Delta^{0}-\odot \Delta\right) \odot \delta \Pi \\
& \left.+\mathrm{i} \int_{\mathcal{C}} \mathrm{d} x \mathrm{~d} y \Pi(x, y) \delta \Delta(y, x)\right\}+\delta \Phi\left\{\phi, \phi^{*}, \Delta, \lambda\right\} .
\end{aligned}
$$

Here $\delta \Pi$ is understood as a variation induced by $\delta \Delta, \delta \phi, \delta \phi^{*}$, and $\delta \lambda$, respectively. In terms of $\Gamma\left\{\phi, \phi^{*}, \Delta, \lambda\right\}$ equations of motion (2.12) and (2.15) read

$$
\delta \Gamma / \delta \phi=0, \delta \Gamma / \delta \phi^{*}=0, \quad \delta \Gamma / \delta \Delta=0
$$

i.e. the functional variations of $\Gamma$ with respect to $\Delta, \phi$ and $\phi^{*}$ at $\lambda(x)=1$ vanish for the physical solutions. These imply the following variational rules for the auxiliary $\Phi$-functional:

$$
\begin{aligned}
\delta \Phi\left\{\phi, \phi^{*}, \Delta, \lambda\right\}=\kappa\left\{\int_{\mathcal{C}} \mathrm{d} x\right. & {\left[J^{*}(x) \delta \phi(x)+J(x) \delta \phi^{*}(x)\right] } \\
& \left.-\mathrm{i} \int_{\mathcal{C}} \mathrm{d} x \mathrm{~d} y \Pi(x, y) \delta \Delta(y, x)\right\}-\int_{\mathcal{C}} \mathrm{d} x \mathcal{E}^{\mathrm{int}}(x) \delta \lambda(x),
\end{aligned}
$$

or

$$
\begin{aligned}
& \mathrm{i} J(x)=\frac{\delta \mathrm{i} \Phi}{\delta \phi^{*}(x)}, \quad-\mathrm{i} \Pi(x, y)=\frac{\delta \mathrm{i} \Phi}{\delta \mathrm{i} \Delta(y, x)} \times \begin{cases}2 & \text { for real fields } \\
1 & \text { for complex fields }\end{cases} \\
& -\mathcal{E}^{\mathrm{int}}(x)=\frac{\delta \mathrm{i} \Phi}{\delta \mathrm{i} \lambda(x)}
\end{aligned}
$$


Thus, $\Phi$ is a generating functional for the source terms $J$ of classical fields and self-energies $\Pi$. Therefore, approximation schemes can be defined through a particular approximation to $\Phi$. The invariance properties of $\Phi$ play a central role to define conservation laws for the approximate dynamics.

It is important to emphasize that we do all functional variations independently on any place of the contour. Thus, different contour times are considered as independent even though they may refer to the same physical time. The fact that components of $\Delta$ on the different branches of the contour are not independent (cf. (A.2) for the physical solution, has no importance for the variational procedure. The reason is that rules (A.2) only apply to the physical $\Delta$ and $\phi$, which are provided by the stationary "points" of the variational principle, i.e. solving the equations of motion (2.12), 2.15). Moreover, for the closed real-time contour the values of $\Gamma$ and $\Phi$ trivially vanish, i.e. $\Gamma=\Phi=0$ for physical values of $\Delta, \phi, \phi^{*}$ and $\lambda=1$.

\section{Diagrams for $\Gamma, \Phi$ and $\mathcal{E}_{\lambda}^{\text {int }}(x)$}

According to (2.21) and (2.25)

$$
-\int_{\mathcal{C}} \mathrm{d} x \mathcal{E}^{\mathrm{int}}(x)=\left[\lambda \frac{\mathrm{d}}{\mathrm{d} \lambda} \Gamma\left\{\phi\{\lambda\}, \phi^{*}\{\lambda\}, \Delta\{\lambda\}, \lambda\right\}\right]_{\lambda=1}=\left[\lambda \frac{\partial}{\partial \lambda} \Phi\left\{\phi, \phi^{*}, \Delta, \lambda\right\}\right]_{\lambda=1},
$$

where now $\lambda$ is considered as a global scale parameter (to $\Phi$ only a partial derivative is applied, i.e. the $\phi, \phi^{*}$ and $\Delta$ values are kept constants). The expression for $-\mathrm{i} \mathcal{E}^{\text {int }}(x)$ can be re-summed and entirely expressed in terms of full classical fields and full propagators. The re-summed diagrams are then void of any self-energy insertions and therefore have to be two-particle irreducible

$$
-\mathrm{i} \mathcal{E}^{\mathrm{int}}(x)=\sum_{n_{\lambda}} \bigoplus_{2 \mathrm{PI}},
$$

i.e. they cannot be decomposed into two pieces by cutting two propagator lines. The formal integration of the last equality in (2.26) with respect to $\lambda$ keeping $\phi$ and $\Delta$ constant provides the diagrammatic expression for $\Phi$ in terms of full Green's functions and classical fields. Therefore i $\Gamma\left\{\phi, \phi^{*}, \Delta, \lambda\right\}$ can be expressed in terms of diagrams (cf. eq. $(2.19)$ as

$$
\mathrm{i} \Gamma\left\{\phi, \phi^{*}, \Delta, \lambda\right\}=\mathrm{i} \Gamma^{0}\left\{\Delta^{0}\right\}+\mathrm{i} \int_{\mathcal{C}} \mathrm{d} x \mathcal{L}^{0}\left\{\phi, \partial_{\mu} \phi\right\}
$$

Here $n_{\Pi}$ counts the number of $\Pi$ insertions in the ring diagrams providing the ln-terms, while for the closed diagrams of $\Phi$ the value $n_{\lambda}$ counts the number of vertices building up the functional $\Phi$. The diagrams contributing to $\Phi$ are given in terms of full propagators $\Delta$ and full time-dependent classical fields $\phi$. As a consequence, these diagrams have to be two-particle irreducible. The latter property is required because of the re-summations of $\mathcal{E}^{\text {int }}(x)$. This also matches diagrammatic rules for the re-summed self-energy $\Pi(x, y)$, which results from functional variation of $\Phi$ with respect to any propagator $\Delta(y, x)$. In graphical terms, this variation is realized by opening a propagator line in all diagrams of $\Phi$. The resulting set of thus opened diagrams must be that of proper skeleton diagrams of $\Pi$ in terms of full propagators, i.e. void of any self-energy insertion.

The diagrammatic rules for $\Phi, \mathcal{E}^{\text {int }}(x), J$ and $\Pi$ are as in perturbation theory, except that (i) for all bosonic fields in $\mathrm{i} \widehat{\mathcal{L}}^{\text {int }}$, replace $\widehat{\phi}$ by $\phi+\widehat{\varphi}$ in order to account for the classical fields; (ii) all pair contractions represent full propagators i $\Delta(x, y)$; (iii) keep only those diagrams that correspond to two-particle irreducible diagrams for $\Phi$, i.e. which cannot be split into two pieces by cutting two different propagator lines. For the rules in the $\{-+\}$ matrix notation we refer to [5]. 
As an example, we quote the diagrams in neutral scalar $g \widehat{\phi}^{4} / 4$ ! theory. Up to two vertices, the functional $\Phi$ is given by the following expressions

$$
\begin{aligned}
\mathrm{i} \Phi & =\frac{-\mathrm{i} g}{4 !} \int_{\mathcal{C}} \mathrm{d} x\left(\phi^{4}(x)+6 \phi^{2}(x)\langle\widehat{\varphi}(x) \widehat{\varphi}(x)\rangle_{c}+3\langle\widehat{\varphi}(x) \widehat{\varphi}(x)\rangle_{c}^{2}\right) \\
& +\frac{1}{2}\left(\frac{-\mathrm{i} g}{4 !}\right)^{2} \int_{\mathcal{C}} \mathrm{d} x \int_{\mathcal{C}} \mathrm{d} y\left(4 \cdot 4 ! \phi(x) \phi(y)\langle\widehat{\varphi}(x) \widehat{\varphi}(y)\rangle_{c}^{3}+4 !\langle\widehat{\varphi}(x) \widehat{\varphi}(y)\rangle_{c}^{4}\right)+\ldots,
\end{aligned}
$$

or in terms of diagrams

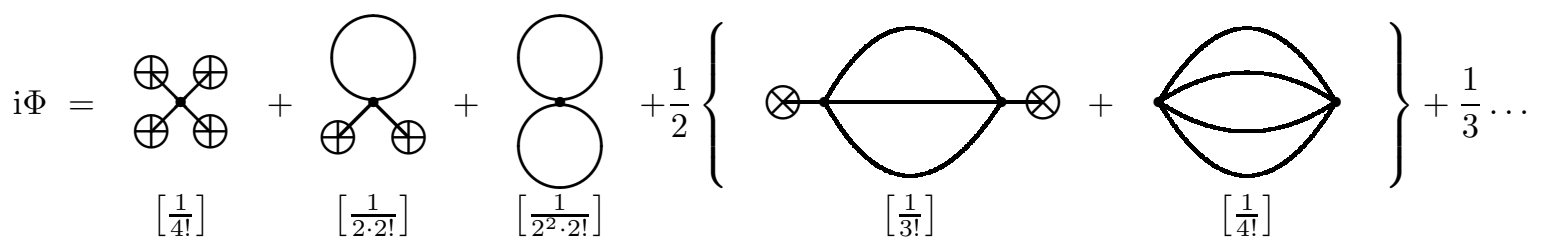

The $1 / n_{\lambda}$ factors are explicitly given, while the standard combinatorial factors are given in square brackets below each diagram. Functional derivatives with respect to $\phi$ (pins) and propagators (full lines), cf. eqs. (2.23), (2.24), determine the source $J(x)$ of the classical field and the self-energy $\Pi(x, y)$, respectively,

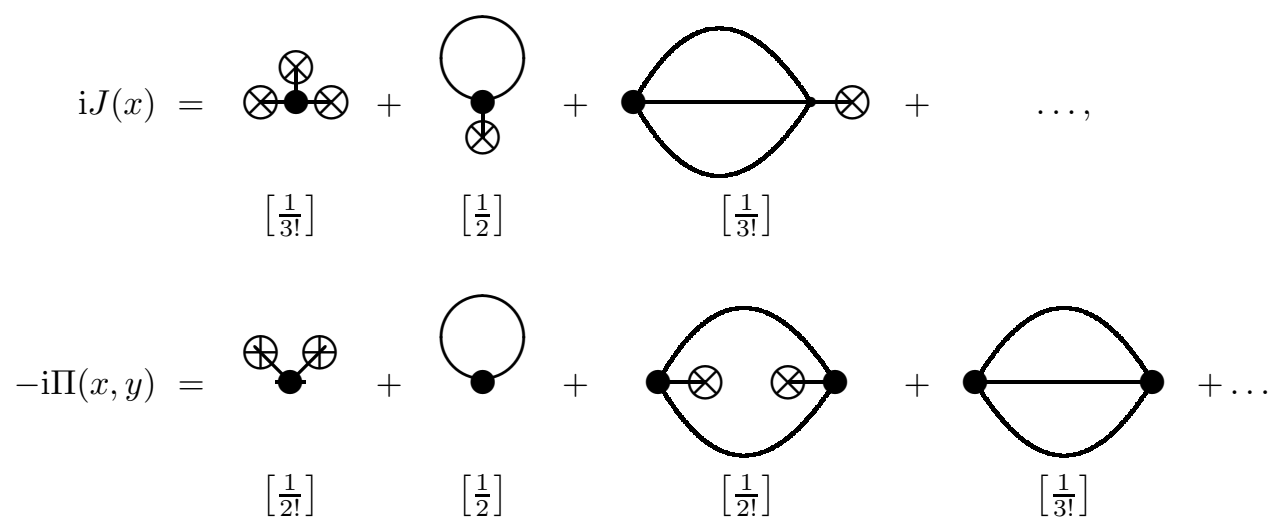

Small full dots define vertices, which are to be integrated over, while big full dots specify the external points $x$ or $y$; the first two diagrams of $\Pi(x, y)$ give the singular $\delta_{\mathcal{C}}(x, y)$ parts arizing from classical fields and tad-poles.

\section{E. $\Phi$-Derivable Approximations and Invariances of $\Phi$}

The expressions for $\Gamma$ and $\Phi$ given above are exact and expressed in terms of full propagators and self-energies. The $\Phi$-derivable approximations [35] are constructed by confining the infinite diagrammatic series for $\Phi$ either to a set of a few diagrams or some sub-series of diagrams. Thereby the approximate $\Phi^{(\text {appr.) }}$ is constructed in terms of full Green's functions and full classical fields, where full now takes the sense of solving self-consistently the classical field and Dyson's equation with the driving terms derived from $\Phi^{\text {(appr.) }}$ through relations (2.24). It means that even restricting ourselves to a single diagram in $\Phi^{\text {(appr.) }}$, in fact, we deal with a whole sub-series of perturbative diagrams. $\Phi^{\text {(appr.) }}$ serves as a generating functional for the approximate source currents $J^{\text {(appr.) }}(x)$ and self-energies $\Pi^{\text {(appr.) }}(x, y)$ (see eqs. (2.24) )

$$
\begin{gathered}
\mathrm{i} J^{(\text {appr. })}(x)=\frac{\delta \mathrm{i} \Phi^{(\text {appr. })}}{\delta\left(\phi^{(\text {appr. }) *(x))}\right.} \\
-\mathrm{i} \Pi^{(\text {appr. })}(x, y)=\frac{\delta \mathrm{i} \Phi^{(\text {appr. })}}{\delta \mathrm{i} \Delta^{(\text {appr. })}(y, x)} \times \begin{cases}2 & \text { for neutral fields } \\
1 & \text { for charged fields }\end{cases}
\end{gathered}
$$

which then are the driving terms for the equations of motion for the classical fields and propagators. $\Phi^{(\text {appr.) }}$ also provides the corresponding expression for $\mathcal{E}^{\text {int }}$ (see eq. (2.25)). Below, we omit the superscript "appr.". 
The invariances of $\Phi$ play as central role as the invariances of the Lagrangian for the full theory. Thereby, the variational principle, where the interaction strength $\lambda(x)$, the classical fields $\phi(x)$ and propagators $\Delta(x, y)$ can be varied independently, provides a set of useful identities and relations. A general invariance of $\Phi$ is provided by the substitution $x \Rightarrow x+\xi(x)$ for all integration variables in the contour integrations defining $\Phi$. This invariance results in the energy-momentum conservation, $\partial_{\mu} \Theta^{\mu \nu}(x)=0$, with the energy-momentum tensor given by the Noether expression

$$
\begin{aligned}
\Theta^{\mu \nu}= & \frac{1}{2} \kappa\left[\left(\left(\partial_{x}^{\nu}\right)^{*}+\partial_{y}^{\nu}\right)\left(\left(\partial_{x}^{\mu}\right)^{*}+\partial_{y}^{\mu}\right)\left(\phi^{*}(x) \phi(y)+\mathrm{i} \Delta(y, x)\right)\right]_{x=y} \\
& +g^{\mu \nu}\left(\mathcal{E}^{\text {int }}(x)-\mathcal{E}^{\text {pot }}(x)\right)
\end{aligned}
$$

with the interaction energy density (2.25) and $\mathcal{E}^{\text {pot }}$ expressed as

$$
\begin{aligned}
\mathcal{E}^{\text {pot }}(x) & =\frac{1}{2} \kappa\left\{-\left[J^{*}(x) \phi(x)+J(x) \phi^{*}(x)\right]\right. \\
& \left.+\mathrm{i} \int_{\mathcal{C}} \mathrm{d} z[\Pi(x, z) \Delta(z, x)+\Delta(x, z) \Pi(z, x)]\right\} .
\end{aligned}
$$

Along similar lines charge conservation can be proven, provided $\Phi$ is invariant under the simultaneous variation of classical fields and propagators

$$
\phi(x) \Rightarrow e^{-\mathrm{i} e \Lambda(x)} \phi(x), \quad \phi^{*}(x) \Rightarrow e^{\mathrm{i} e \Lambda(x)} \phi^{*}(x), \quad \Delta(x, y) \Rightarrow e^{-\mathrm{i} e \Lambda(x)} \Delta(x, y) e^{\mathrm{i} e \Lambda(y)} .
$$

By means of equations of motion (2.12), (2.15) the divergence of the Noether current

$$
j^{\mu}(x)=e\left[\left(\left(\partial_{x}^{\mu}\right)^{*}+\partial_{y}^{\mu}\right)\left(\phi^{*}(x) \phi(y)+\mathrm{i} \Delta(y, x)\right)\right]_{x=y}
$$

vanishes. Thus, the current conservation takes place for any $\Phi$-derivable approximation, which is invariant with respect to (2.36).

Further invariances generally depend on the properties of the interaction vertices in the theory considered. An example is the invariance discussed in the context of eq. (2.7), which now transcribes to the corresponding expectation values.

\section{F. Generating Functional $\Gamma^{\mathrm{eq}}$ and Thermodynamic Consistency}

In the thermal equilibrium the thermodynamic potential is explicitly known, cf. [37],

$$
\Omega=-T \ln Z, \quad \text { where } \quad \widehat{\rho}^{\mathrm{eq}}=\frac{\exp (-\beta \widehat{H}\{\mu\})}{Z}, \quad \text { with } \quad \operatorname{Tr} \widehat{\rho}^{\mathrm{eq}}=1,
$$

where $\beta=1 / T$ is the inverse temperature, $\widehat{\rho}^{\mathrm{eq}}$ is the equilibrium density operator and $Z$ is the partition function,

$$
\widehat{H}(\mu)=\widehat{H}-\int \mathrm{d}^{3} x \mu \widehat{j}_{(\text {Noether })}^{0}(x),
$$

where $\widehat{j}_{\text {(Noether) }}^{0}$ is the time-component of the charge current, $\mu$ is the chemical potential.

The equilibrium density matrix formally coincides with evolution operator in imaginary time, i.e. one writes Tr $\widehat{\rho}^{\text {eq }} \ldots$ instead of $\langle\ldots\rangle$. Thus, we arrive at the following form of $\Gamma$ functional in equilibrium:

$$
\Gamma^{\mathrm{eq}}\left\{\phi, \phi^{*}, \Delta, \lambda, \mu\right\}=-\mathrm{i} \ln \left(\frac{1}{Z} \operatorname{Tr} \exp \left[-\mathrm{i} \int_{\mathcal{C}} \mathrm{d} t \widehat{H}_{\mathrm{I}}^{0}\{\mu\}\right] \mathcal{T}_{\mathcal{C}} \exp \left[\mathrm{i} \int_{\mathcal{C}} \mathrm{d} x \lambda \widehat{\mathcal{L}}_{\mathrm{I}}^{\mathrm{int}}\{\mu\}\right]\right),
$$

with the integration contour $\mathcal{C}$ now being the sum of the real-time Schwinger-Keldysh contour (see figure) and the imaginary-time Matsubara contour $\mathcal{C}_{\text {eq }}$, i.e. it starts from an initial time $t_{0}$, goes to infinity, then back to this initial time and after that, to $t_{0}-\mathrm{i} \beta$. Taking into account the fact that $\Gamma=0$ for the physical values of $\phi, \phi^{*}, \Delta$, and $x$-independent $\lambda$, from eq. (2.38) we obtain

$$
\Omega\left\{\phi, \phi^{*}, \Delta, \lambda, \mu\right\}=-T \ln \left\{\operatorname{Tr}\left(\exp \left[-\mathrm{i} \int_{\mathcal{C}_{\text {eq }}} \mathrm{d} t \widehat{H}_{\mathrm{I}}^{0}\{\mu\}\right] \mathcal{T}_{\mathcal{C}} \exp \left[\mathrm{i} \int_{\mathcal{C}_{\mathrm{eq}}} \mathrm{d} x \lambda \widehat{\mathcal{L}}_{\mathrm{I}}^{\mathrm{int}}\{\mu\}\right]\right)\right\} .
$$


where the integral over the real-time contour section gives zero. In eq. (2.41) we can make the replacement

$$
\int_{\mathcal{C}_{\text {eq }}} \mathrm{d} t \ldots=\int_{0}^{-\mathrm{i} \beta} \mathrm{d} t \ldots
$$

Thus, one arrives at the proper representation of the thermodynamic potential originally proposed by Luttinger and Ward [36]. Indeed, since all quantities under the integral are analytically continued from the Schwinger-Keldysh contour to the Matsubara contour, $\Omega$ is determined by the same expression as the $\Gamma$-functional (2.19) but in terms of the Matsubara Green's functions with the thermodynamic $\Phi_{T}$-functional represented by the same set of closed diagrams. Thus, the problem of the thermodynamic consistency is re-addressed from the Schwinger-Keldysh approach to the Matsubara one. Within the Matsubara formalism, ref. [35] has shown that any $\Phi$-derivable approximation to the thermodynamic potential is thermodynamically consistent. Hence, the $\Phi$-derivable approximations to non-equilibrium $\Gamma$-functional are also thermodynamically consistent [12].

The stationary property of the $\Gamma$ functional (and, hence, of $\Omega$ ) with respect to variations in full Green's functions and classical fields, eq. (2.22), is the key feature of $\Omega$ that provides the thermodynamic consistency. It implies that any derivative of the partition sum to any thermodynamic parameter like $\beta$ or $\mu$ is then given by accounting only the explicit dependence of $\Omega$ on these parameters, since the implicit dependences through $\Delta$ and $\phi$ drop out due to the stationarity. Therefore $\Phi$-derivable approximations preserve the corresponding properties of the exact theory, providing thermodynamic consistency.

\section{NON-EQUILIBRIUM AND THERMODYNAMIC POTENTIALS}

It is instructive to introduce a non-equilibrium potential $\widetilde{\Omega}$ and to get the relation between this potential, the $\Gamma$ functional introduced in eq. (2.19), and the thermodynamical potential $\Omega$ (in thermal equilibrium). This relation will provide another and more direct way for demonstrating thermodynamic consistency of $\Phi$-derivable approximations in the spirit of Baym [35] and also can be useful in certain applications of the $\Phi$-derivable approach.

For the sake of convenience, we introduce the zero component of the operator $\widehat{p}_{x}^{0}$ shifted by the chemical potential

$$
\widehat{\pi}_{x}^{0}=\mathrm{i}\left(\partial_{t}-\mathrm{i} e \mu,-\nabla_{x}\right) .
$$

We have introduced the chemical potential in such a way that it fixes a conserving quantity, related to some charge (e.g., electric charge, strangeness, etc.).

Let us introduce an auxiliary non-equilibrium potential $\widetilde{\Omega}$ :

$$
\Gamma\left\{\phi, \phi^{*}, \Delta, \lambda, \mu\right\}=-\int_{\mathcal{C}} \mathrm{d} t \widetilde{\Omega}\left\{\phi, \phi^{*}, \Delta, \lambda, \mu\right\} .
$$

Unlike $\Gamma$, the new quantity $\widetilde{\Omega}$ is non-zero for physical values of Green's functions and mean fields. The value $-\widetilde{\Omega}$ plays the role of the effective Lagrangian. Below we show that in thermal equilibrium this non-equilibrium potential has the meaning of the usual thermodynamic potential. In order to demonstrate this, we first find variations of $\widetilde{\Omega}$ with respect to the chemical potential and to the $\lambda$-vertex scaling parameter at arbitrary non-equilibrium. Based on the identity (3.2), we can extend the method of the on-contour variations of $\Gamma$ in order to get derivatives of non-equilibrium and thermodynamic potentials.

The $\Gamma$-functional introduced above is now a functional of the chemical potential. This functional dependence can be treated in two ways. First, we can consider the functional dependence of $\Gamma$ on $\mu$ as originated solely from changes of Green's functions, self-energies and mean fields, which are induced by a variation of the chemical potential. Indeed, the additional term of eq. (2.39) can be completely absorbed into equations of motion by changing $\partial_{t} \rightarrow \partial_{t}-\mathrm{i} e \mu$ in $S_{x}$ operators of eq. (2.2). Notice that now the free Green's functions (cf. eq. (2.14)) also depend on the chemical potential rather than only full Green's functions and self-energies.

Let us calculate the variation of $\Gamma$ with respect to $\delta \mu$ in the above-described way, provided all other variables of the $\Gamma$-functional are kept constant. Here, we allow the variation of $\mu$ on the contour, i.e., $\delta \mu(x)$ is a contour function, which, in general, takes different values on different branches of the contour. To get the variation $\delta \Delta^{0}(x, y)$ induced by $\delta \mu(x)$, we vary eq. (2.14). Then we obtain

$$
S_{x}(\mu) \delta \Delta^{0}(x, y)=-\delta S_{x}(\mu) \Delta^{0}(x, y)
$$

where

$$
\delta S_{x}(\mu)=-e\left(\delta \mu(x) \widehat{\pi}_{x}^{0}+\widehat{\pi}_{x}^{0} \delta \mu(x)\right)
$$


or, by using the fact that $\Delta^{0}$ is the resolvent of the $S_{x}$ operator, we arrive at

$$
\begin{aligned}
\delta \Delta^{0}(x, y) & =-\int_{\mathcal{C}} \mathrm{d} z \Delta^{0}(x, z) \delta S_{z}(\mu) \Delta^{0}(z, y) \\
& =-\int_{\mathcal{C}} \mathrm{d} z \delta \mu(z) e\left(\Delta^{0}(x, z)\left[\widehat{\pi}_{z}^{0} \Delta^{0}(z, y)\right]-\left[\widehat{\pi}_{z}^{0} \Delta^{0}(x, z)\right] \Delta^{0}(z, y)\right) .
\end{aligned}
$$

To calculate variations with respect to $\Delta^{0}$ we need the rule to vary $\Gamma^{0}$ :

$$
\delta \Gamma^{0}=-\mathrm{i} \kappa \int_{\mathcal{C}} \mathrm{d} x\left(S_{x} \delta \Delta^{0}(x, y)\right)_{y=x},
$$

which follows from the definition of $\Gamma^{0}$ (cf. eq. (2.19) $)$. Varying now the free part of $\Gamma$ in $\mu$ according to eq. (3.6), we obtain

$$
\delta \Gamma^{0}=\mathrm{i} \kappa e \int_{\mathcal{C}} \mathrm{d} x\left[\left(\widehat{\pi}_{x}^{0}+\left(\widehat{\pi}_{y}^{0}\right)^{*}\right) \Delta^{0}(x, y)\right]_{y=x} \delta \mu(x) .
$$

The interaction part of $\Gamma$ (cf. eq. (2.19) ) depends on $\mu$ only through $\Delta, \Pi, \Delta^{0}$, mean fields and their derivatives. Note that $\Gamma$ is stationary under variations in $\Delta$ and mean fields near their physical values. Hence, the variation of $\left(\Gamma-\Gamma^{0}\right)$ results only from the variations of $\Delta^{0}$ and mean-field derivatives in $\mathcal{L}^{0}\left\{\phi, \partial_{\mu} \phi\right\}$. Thus, varying $\left(\Gamma-\Gamma^{0}\right)$, we get (in symbolic form of eq. (2.19))

$$
\begin{aligned}
& \delta\left(\Gamma-\Gamma^{0}\right)=\kappa e \int_{\mathcal{C}} \mathrm{d} x\left[\phi^{*} \cdot \widehat{\pi}_{x}^{0} \phi+\left(\widehat{\pi}_{x}^{0}\right)^{*} \phi^{*} \cdot \phi\right] \delta \mu(x) \\
& -\mathrm{i} \kappa \odot \frac{1}{\left(1-\odot \Delta^{0} \odot \Pi\right)} \odot \delta \Delta^{0} \odot \Pi .
\end{aligned}
$$

Substituting here $\delta \Delta^{0}$ in the form of eq. (3.5), we arrive at

$$
\begin{aligned}
\delta\left(\Gamma-\Gamma^{0}\right) & =\kappa e \int_{\mathcal{C}} \mathrm{d} x\left[\phi^{*} \cdot \widehat{\pi}_{x}^{0} \phi+\left(\widehat{\pi}_{x}^{0}\right)^{*} \phi^{*} \cdot \phi\right] \delta \mu(x) \\
& +\mathrm{i} \kappa e \int_{\mathcal{C}} \mathrm{d} x \mathrm{~d} z \mathrm{~d} z^{\prime}\left[\left(\widehat{\pi}_{x}^{0}+\left(\widehat{\pi}_{y}^{0}\right)^{*}\right) \Delta^{0}(x, z) \Pi\left(z, z^{\prime}\right) \Delta\left(z^{\prime}, y\right)\right]_{y=x} \delta \mu(x) .
\end{aligned}
$$

It is important to point out that we have essentially used the variation rules (2.23) to get this expression. Thus, the final result is

$$
\begin{aligned}
\delta \Gamma & =\int_{\mathcal{C}} \mathrm{d} x \delta \mu(x) \kappa e\left(\left[\phi^{*} \cdot \widehat{\pi}_{x}^{0} \phi+\left(\widehat{\pi}_{x}^{0}\right)^{*} \phi^{*} \cdot \phi\right]\right. \\
& \left.+\mathrm{i}\left[\left(\widehat{\pi}_{x}^{0}+\left(\widehat{\pi}_{y}^{0}\right)^{*}\right) \Delta(x, y)\right]_{y=x}\right) .
\end{aligned}
$$

Comparing the expression under the integral with that for the current (2.37), we see that the above variation takes the form

$$
\delta \Gamma\left\{\phi\{\mu\}, \phi^{*}\{\mu\}, \Delta\{\mu\}, \lambda=\text { const, } \mu(x)\right\}=\int_{\mathcal{C}} \mathrm{d} x j^{0}(x) \delta \mu(x),
$$

where $j^{0}$ is the zero-component of the current, i.e. the density. In all we have done so far, we did not assume the thermal equilibrium. Hence, eq. (3.11) holds for any non-equilibrium.

If we perform all the same manipulations for the equilibrium $\Gamma$-functional $\left(\Gamma^{\mathrm{eq}}\right)$ defined by eq. (2.40), we arrive at precisely the same result (3.11) but in equilibrium. On the other hand, in equilibrium we can straightforwardly vary eq. (2.40) in the chemical potential and, hence, immediately arrive again at eq. (3.11). The coincidence of these two ways of variation shows that we are able to consistently introduce chemical potentials only provided we deal with a $\Phi$-derivable approximation, i.e. when the variation rule (2.23) holds true.

Now, comparing eq. (3.11) with eq. (3.2), we obtain

$$
\delta \widetilde{\Omega}\{\mu(x)\}=-\int \mathrm{d}^{3} x j^{0}(x) \delta \mu(x) .
$$


This exactly coincides with the respective derivative of the thermodynamic potential $\Omega$ in equilibrium

$$
\frac{\partial \Omega(\mu)}{\partial \mu}=-\int \mathrm{d}^{3} x j^{0} \equiv-N_{e},
$$

thus yielding the total charge $N_{e}$ associated with $j$-current. Therefore, we conclude that

$$
\int \mathrm{d}^{3} x\left(\frac{\delta \widetilde{\Omega}\{\mu\}}{\delta \mu}\right)_{\text {eq }}=\frac{\partial \Omega(\mu)}{\partial \mu} .
$$

We can also find a variation of $\widetilde{\Omega}$ with respect to $\lambda$ being considered a contour function of time. From eqs. (2.21), (2.23) we have

$$
\delta \Gamma\left\{\phi\{\lambda\}, \phi^{*}\{\lambda\}, \Delta\{\lambda\}, \lambda(x), \mu=\mathrm{const}\right\}=-\int_{\mathcal{C}} \mathrm{d} t \int \mathrm{d}^{3} x \mathcal{E}_{\lambda}^{\mathrm{int}}(x) \delta \lambda(x),
$$

or in terms of the non-equilibrium potential

$$
\delta \widetilde{\Omega}\{\lambda\}=\int \mathrm{d}^{3} x \mathcal{E}_{\lambda}^{\mathrm{int}}(x) \delta \lambda(x) .
$$

On the other hand, in statistical physics, cf. [37], there is a well-known theorem relating a variation of the thermodynamical potential $\Omega$ in a parameter $\lambda$ to the statistical average of the corresponding variation of $\widehat{\mathcal{L}}^{\text {int }}$ in the same parameter

$$
\frac{\partial \Omega(\lambda)}{\partial \lambda}=-\int \mathrm{d}^{3} x\left\langle\frac{\partial \widehat{\mathcal{L}}^{\text {int }}}{\partial \lambda}\right\rangle=\int \mathrm{d}^{3} x \mathcal{E}_{\lambda}^{\text {int }}(x) .
$$

With $\lambda$, being the scaling factor of the interaction Lagrangian of eq. (2.20), this relation follows from expression (2.41) for the thermodynamic potential. It is completely similar to eq. 3.16) for the non-equilibrium potential. Thus, we obtain in equilibrium

$$
\int \mathrm{d}^{3} x\left(\frac{\delta \widetilde{\Omega}\{\lambda\}}{\delta \lambda}\right)_{\text {eq }}=\frac{\partial \Omega(\lambda)}{\partial \lambda} .
$$

Hence, proceeding from properties (3.14) and (3.18), we conclude that the above-introduced non-equilibrium potential has the meaning of the thermodynamical potential in equilibrium

$$
\widetilde{\Omega}_{\mathrm{eq}}=\Omega \text {. }
$$

Thus defined $\widetilde{\Omega}$ is an extension of the thermodynamic potential to non-equilibrium processes. In a way, this nonequilibrium potential is equivalent to the $\Gamma$-functional, while the former has the advantage that it is non-zero for physical values of Green's functions and mean fields.

Relation (3.2) between $\Gamma$ and $\widetilde{\Omega}$ allows us to directly evaluate $\widetilde{\Omega}$ in terms of transport quantities. In particular, a useful scheme of calculating the non-equilibrium and thermodynamic potentials consists in integrating the interaction energy over the coupling constant (or $\lambda$, in our case), cf. [37] for equilibrium systems. Indeed, from eq. (3.16) we get

$$
\widetilde{\Omega}=\int \mathrm{d}^{3} x \int_{0}^{1} \mathrm{~d} \lambda \mathcal{E}_{\lambda}^{\mathrm{int}}(x)+\widetilde{\Omega}_{0},
$$

where $\widetilde{\Omega}_{0}$ is a $\lambda$-independent part, corresponding to the system of non-interacting particles. Analogous expression is obtained from eq. (3.17) in the equilibrium case. For specific interactions with a certain number $\gamma$ of operators attached to the vertex, with the help of eq. (2.7) we obtain

$$
\widetilde{\Omega}=\frac{2}{\gamma} \int \mathrm{d}^{3} x \int_{0}^{1} \mathrm{~d} \lambda \mathcal{E}_{\lambda}^{\mathrm{pot}}(x)+\widetilde{\Omega}_{0} .
$$


Applying eq. (2.35) we arrive at the expression

$$
\begin{aligned}
\widetilde{\Omega} & =\frac{2}{\gamma} \int \mathrm{d}^{3} x \int_{0}^{1} \frac{\mathrm{d} \lambda}{\lambda}\left(\frac { 1 } { 2 } \kappa \left\{-\left[\phi^{*}(x) J(x)+J^{*}(x) \phi(x)\right]\right.\right. \\
& \left.\left.+\mathrm{i} \int_{\mathcal{C}} \mathrm{d} z[\Pi(x, z) \Delta(z, x)+\Delta(x, z) \Pi(z, x)]\right\}\right)+\widetilde{\Omega}_{0} .
\end{aligned}
$$

which allows us to evaluate it directly in terms of non-equilibrium transport quantities.

In equilibrium, $\Delta$ and $\Pi$ are given by expressions of eq. (A.6). Then (3.22) transforms into the thermodynamic potential

$$
\begin{aligned}
\Omega & =\frac{2}{\gamma} \int \mathrm{d}^{3} x \int_{0}^{1} \frac{\mathrm{d} \lambda}{\lambda} \kappa\left(-\frac{1}{2}\left[\phi^{*}(x) J(x)+J^{*}(x) \phi(x)\right]\right. \\
& \left.+\int \frac{\mathrm{d}^{4} p}{(2 \pi)^{4}} n\left(p_{0}\right)\left[\operatorname{Re} \Pi^{R}(\lambda) A(\lambda)+\operatorname{Re} \Delta^{R}(\lambda) \Gamma(\lambda)\right]\right)+\Omega_{0},
\end{aligned}
$$

where $A=-2 \operatorname{Im} \Delta^{R}$ and $\Gamma=-2 \operatorname{Im} \Pi^{R}$ are the spectral function and spectral width, respectively, while $n\left(p_{0}\right)$ is the thermal Bose-Einstein occupation number (A.7).

\section{NON-EQUILIBRIUM POTENTIAL, STRESS TENSOR, PRESSURE AND BULK VISCOSITY}

Let us consider $\delta \Gamma$ induced by an infinitesimal scaling transformation of the space on the contour

$$
\boldsymbol{x}^{\prime}=(1+\xi(t)) \boldsymbol{x}, \quad \xi(t) \ll 1 .
$$

This transformation results in a change of the volume of the system

$$
V^{\prime}=\int \mathrm{d}^{3} x^{\prime}=(1+\xi(t))^{3} \int \mathrm{d}^{3} x \simeq(1+3 \xi(t)) V .
$$

Under this transformation the $S_{x}$ operator changes as

$$
\delta S_{x}(\mu)=2 \xi(t) \widehat{\boldsymbol{p}}_{x}^{2},
$$

where $\widehat{\boldsymbol{p}}_{x}$ is the spatial vector related to $\widehat{p}_{x}^{\mu}=-\mathrm{i} \partial^{\mu}$. The variation of the free Green's function is determined by the equation

$$
S_{x} \delta \Delta^{0}(x, y)=-\delta S_{x} \Delta^{0}(x, y)-3 \xi(t) \delta_{\mathcal{C}}(x, y),
$$

where we have taken into account that the scaling transformation (4.1) also modifies the contour $\delta$-function. Using the fact that $\Delta^{0}$ is the resolvent of the $S_{x}$ operator, we get

$$
\delta \Delta^{0}(x, y)=-\int_{\mathcal{C}} \mathrm{d} z \Delta^{0}(x, z) \delta S_{z}(\mu) \Delta^{0}(z, y)-3 \xi(t) \Delta^{0}(x, y) .
$$

Similarly, the scale transformation (4.1) induces variations $\delta \Delta, \delta \Pi, \delta \phi$ and $\delta \phi^{*}$. However, in view of the stationary properties of the $\Gamma$-functional, we do not need explicit forms of these variations. Thus, variation of the $\Gamma$ is determined by the variation of $\Delta^{0}$ and variations of internal integrations entering into the definition of the $\Gamma$-functional, eq. (2.19). For the $\Phi$-functional the variations of internal integrations can be associated with variation of $\lambda$

$$
\int \mathrm{d}^{3} x^{\prime} \ldots=\int \mathrm{d}^{3} x(1+\xi(t))^{3} \ldots=\int \mathrm{d}^{3} x \lambda^{\prime} \ldots
$$

where

$$
\delta \lambda=\lambda^{\prime}-1 \simeq 3 \xi(t) .
$$

Now performing the variation of $\Gamma^{0}$, according to eqs. (3.6), (4.5) and (4.3) we obtain

$$
\delta \Gamma^{0}=\mathrm{i} \kappa \int_{\mathcal{C}} \mathrm{d} x\left[\frac{1}{6}\left(\widehat{\boldsymbol{p}}_{x}+\left(\widehat{\boldsymbol{p}}_{y}\right)^{*}\right)^{2} \Delta^{0}(x, y)\right]_{y=x} 3 \xi(t) .
$$


Note that the contribution $-3 \xi(t) \Delta^{0}$ in the $\Delta^{0}$ variation of eq. 4.5 has exactly cancelled out the contribution emerged from the variation of the internal integration. Let us now turn to the mean-field term, i.e. the second term in (2.19). The variation of this term is induced by the changes of internal integration and mean-field gradients in $\mathcal{L}^{0}$

$$
\frac{\partial}{\partial \boldsymbol{x}^{\prime}} \simeq(1-\xi(t)) \frac{\partial}{\partial \boldsymbol{x}}
$$

as well as by variations of mean fields

$$
\begin{gathered}
\delta\left(\int_{\mathcal{C}} \mathrm{d} x \mathcal{L}^{0}\left\{\phi, \partial_{\mu} \phi\right\}\right)=-\kappa \int_{\mathcal{C}} \mathrm{d} x \frac{1}{2}\left(\phi^{*} J+J^{*} \phi\right) 3 \xi(t) \\
-\kappa \int_{\mathcal{C}} \mathrm{d} x \frac{1}{6}\left[\left(\nabla_{x}-\nabla_{y}\right)^{2} \phi^{*}(x) \phi(y)\right]_{y=x} 3 \xi(t)+O\left(\delta \phi, \delta \phi^{*}\right) .
\end{gathered}
$$

Here the first term on the r.h.s. results from the variation of the internal integration, the second, from the variation of gradient terms, while $O\left(\delta \phi, \delta \phi^{*}\right)$ denotes the contribution of variations of mean fields. We do not present this last term in an explicit form, since any case it is cancelled out by the respective variations of the $\Phi$-functional. This is the stationary property of the $\Gamma$-functional with respect to variations of mean fields near their physical values.

Now consider the main interaction terms in the $\Gamma$-functional. We obtain

$$
\begin{aligned}
& \delta\left(\Gamma-\Gamma^{0}-\int_{\mathcal{C}} \mathrm{d} x \mathcal{L}^{0}\left\{\phi, \partial_{\mu} \phi\right\}\right) \\
= & \mathrm{i} \kappa \int_{\mathcal{C}} \mathrm{d} x \mathrm{~d} z \mathrm{~d} z^{\prime}\left[\frac{1}{6}\left(\widehat{\boldsymbol{p}}_{x}+\left(\widehat{\boldsymbol{p}}_{y}\right)^{*}\right)^{2} \Delta^{0}(x, z) \Pi\left(z, z^{\prime}\right) \Delta\left(z^{\prime}, y\right)\right]_{y=x} 3 \xi(t) \\
+ & \mathrm{i} \frac{1}{2} \kappa \int_{\mathcal{C}} \mathrm{d} x \mathrm{~d} z[\Delta(x, z) \Pi(z, x)+\Pi(x, z) \Delta(z, x)] 3 \xi(t) \\
- & \int_{\mathcal{C}} \mathrm{d} x \mathcal{E}^{\operatorname{int}}(x) 3 \xi(t)-O\left(\delta \phi, \delta \phi^{*}\right) .
\end{aligned}
$$

Here the first two terms on the r.h.s. of eq. (4.11) result from the $\delta \Delta^{0}$ in the ln-term of $\Gamma$, these are derived very similarly to that in eq. (3.9) by using eqs. (4.5) and (4.3) for the $\delta \Delta^{0}$ variation. The third term comes from the variation of internal integrations (4.2) in the $\Phi$-functional. Deriving it, we used the fact that the variation of internal integrations in the $\Phi$-functional is equivalent to its variation in $\lambda$ (cf. eqs. (4.6) and (4.7)). The term $O\left(\delta \phi, \delta \phi^{*}\right)$ denotes the contribution of variations of mean fields, which is explicitly cancelled out by the respective variations in eq. (4.10).

Collecting all the above terms of eqs. (4.8), (4.10) and (4.11) and using Dyson's equation (2.15), we arrive at

$$
\begin{aligned}
\delta \Gamma & =\int_{\mathcal{C}} \mathrm{d} x 3 \xi(t)\left[\frac{1}{6} \mathrm{i} \kappa\left[\left(\widehat{\boldsymbol{p}}_{x}+\left(\widehat{\boldsymbol{p}}_{y}\right)^{*}\right)^{2} \Delta(x, y)\right]_{y=x}\right. \\
& \left.-\frac{1}{6} \kappa\left[\left(\nabla_{x}-\nabla_{y}\right)^{2} \phi^{*}(x) \phi(y)\right]_{y=x}-\left(\mathcal{E}^{\mathrm{int}}(x)-\mathcal{E}^{\mathrm{pot}}(x)\right)\right] .
\end{aligned}
$$

Comparing the r.h.s. of this expression with that for the energy-momentum tensor (2.34) and taking account of eq. (4.2), we see that

$$
\delta \Gamma=\int_{\mathcal{C}} \mathrm{d} t \frac{1}{3} \Theta^{i i} \delta V(t)
$$

with the summation over the repeated Latin indices $i=1,2,3$. Thus,

$$
\delta \widetilde{\Omega}=-\frac{1}{3} \Theta^{i i} \delta V(t)
$$

Combining (3.12) and (4.14) we arrive at the expression

$$
\widetilde{\Omega}=-\frac{1}{3} \int \mathrm{d}^{3} x \Theta^{i i}
$$

Note that deriving above results we have not assumed anywhere the thermal equilibrium. Therefore, they hold for arbitrary non-equilibrium. 
In equilibrium, in the rest frame, where collective velocity of the matter is zero, $\Theta^{i i} / 3=P_{\text {eq }}\left(P_{\text {eq }}\right.$ is the equilibrium pressure of the matter). Therefore, we can associate $\widetilde{\Omega}$ with a non-equilibrium pressure $\widetilde{P}$ :

$$
\widetilde{\Omega}=-\int \mathrm{d}^{3} x \widetilde{P},
$$

of course, in the local rest frame of the matter. In general, local rest frames are different at different $\mathbf{x}$. Therefore, here and below integration over $\mathrm{d}^{3} x$ should be understood as the integration over small volume around $\mathbf{x}$.

Slightly out of equilibrium the energy-momentum tensor takes the form [55]

$$
\begin{aligned}
\Theta^{\mu \nu} & =\left(\varepsilon_{\mathrm{eq}}+P_{\mathrm{eq}}\right) u^{\mu} u^{\nu}-g^{\mu \nu} P_{\mathrm{eq}}+\pi^{\mu \nu} \\
\pi^{\mu \nu} & =\eta\left(\partial^{\mu} u^{\nu}+\partial^{\nu} u^{\mu}-u^{\mu} u_{\lambda} \partial^{\lambda} u^{\nu}-u^{\nu} u_{\lambda} \partial^{\lambda} u^{\mu}\right)+\left(\zeta-\frac{2}{3} \eta\right)\left(g^{\mu \nu}-u^{\mu} u^{\nu}\right) \partial_{\lambda} u^{\lambda}
\end{aligned}
$$

where $\varepsilon_{\text {eq }}$ and $P_{\text {eq }}$ are local equilibrium proper energy density and pressure, respectively, and $u_{\mu}$ is a local 4 -velocity. All these quantities are, in general, $x$ dependent. Non-equilibrium effects are associated with $\pi^{\mu \nu}$ tensor, i.e. with shear $(\eta)$ and bulk $(\zeta)$ viscosities. Here we put aside various causal extensions of dissipative hydrodynamics [59]. Then the non-equilibrium potential takes the form

$$
\widetilde{\Omega}=-\frac{1}{3} \int \mathrm{d}^{3} x\left[\left(\varepsilon_{\mathrm{eq}}+P_{\mathrm{eq}}\right) u^{i} u^{i}+3 P_{\mathrm{eq}}+2 \eta\left(\partial^{i} u^{i}-u^{i} u_{\lambda} \partial^{\lambda} u^{i}\right)-\left(\zeta-\frac{2}{3} \eta\right)\left(3+u^{i} u^{i}\right) \partial_{\lambda} u^{\lambda}\right] .
$$

In the local rest frame, i.e. at $\mathbf{u}=0$, it reads

$$
\widetilde{\Omega}=-\int \mathrm{d}^{3} x\left[P_{\mathrm{eq}}-\zeta \partial_{i} u^{i}\right],
$$

which is a key relation for evaluation of $\zeta$. To obtain the latter equality we used the fact that the term $\propto \partial_{0} u^{0}$ in eq. (4.20) should be omitted because $\partial_{0} u^{0} \propto u^{i} \partial_{0} u^{i}$. Since $\zeta>0$ for stable systems, the non-equilibrium contribution to the pressure (4.20) is negative for expanding system (for $\partial_{i} u^{i}>0$ ) and positive for contracting system (for $\partial_{i} u^{i}<0$ ), see [19].

It is reasonable to use the following setup in order to evaluate the bulk viscosity. Proceeding from certain $\Phi$ functional, a problem of Hubble-like expansion of the matter should be solved within the non-equilibrium Green's function technique. The advantage of the Hubble-like setup is that the system remains spatially homogeneous during the expansion. The "rate" of expansion, $\partial_{i} u^{i}$, is an external parameter, that can be prescribed any value because the problem does not contain any spatial scale. Initial conditions for this expansion should be chosen in such a way that deviations from the corresponding equilibrium solution are small. A possible choice is the equilibrium solution boosted in accordance with the Hubble rule. This boosted equilibrium initial condition still presents a locally equilibrium configuration. Genuine non-equilibrium develops only during the expansion governed by real-time-contour equations of motion. Upon solving this problem, the non-equilibrium pressure $\widetilde{P}=P_{\mathrm{eq}}-\zeta \partial_{i} u^{i}$ can be determined in terms of 2PI diagrams according to rules formulated in subsect. IID The equilibrium pressure $P_{\text {eq }}$ should be determined within a thermodynamic calculation based on the same $\Phi$ functional. Then, the bulk viscosity can be directly determined.

\section{SUMMARY}

We constructed a non-equilibrium potential, associated with a pressure for non-equilibrium systems and formulated the rules for its calculation within the $\Phi$-functional method. The non-equilibrium potential transforms to the ordinary thermodynamic potential in case of the local equilibrium and equilibrium systems. By means of variations of this nonequilibrium potential over respective parameters it is possible to calculate the same quantities in the non-equilibrium as those with the help of the thermodynamic potential. Since our non-equilibrium potential can be expressed in terms of 2PI diagrams within the non-equilibrium Green's function technique on the Schwinger-Keldysh contour, it opens new possibilities of evaluating these quantities. In particular, a possible application of this method to calculation of the bulk viscosity is described.

\section{Acknowledgements}

We are grateful to J. Knoll for numerous discussions and valuable remarks. Y.B.I. was partially supported by the grant NS-215.2012.2. 


\section{Appendix A: Contour Function Relations}

Due to the change of operator ordering genuine multi-point functions are discontinuous in general, when two contour coordinates become identical. In particular, two-point functions like i $F(x, y)=\left\langle\mathcal{T}_{\mathcal{C}} \widehat{A}(x) \widehat{B}(y)\right\rangle$ become

$$
\mathrm{i} F(x, y)=\left(\begin{array}{cc}
\mathrm{i} F^{--}(x, y) & \mathrm{i} F^{-+}(x, y) \\
\mathrm{i} F^{+-}(x, y) & \mathrm{i} F^{++}(x, y)
\end{array}\right)=\left(\begin{array}{cc}
\langle\mathcal{T} \widehat{A}(x) \widehat{B}(y)\rangle & \langle\widehat{B}(y) \widehat{A}(x)\rangle \\
\langle\widehat{A}(x) \widehat{B}(y)\rangle & \left\langle\mathcal{T}^{-1} \widehat{A}(x) \widehat{B}(y)\right\rangle
\end{array}\right),
$$

where $\mathcal{T}$ and $\mathcal{T}^{-1}$ are the usual time and anti-time ordering operators. From eq. A.1 follow relations between non-equilibrium and usual retarded and advanced functions

$$
\begin{aligned}
F^{R}(x, y) & =F^{--}(x, y)-F^{-+}(x, y)=F^{+-}(x, y)-F^{++}(x, y) \\
& :=\Theta\left(x_{0}-y_{0}\right)\left(F^{+-}(x, y)-F^{-+}(x, y)\right), \\
F^{A}(x, y) & =F^{--}(x, y)-F^{+-}(x, y)=F^{-+}(x, y)-F^{++}(x, y) \\
& :=-\Theta\left(y_{0}-x_{0}\right)\left(F^{+-}(x, y)-F^{-+}(x, y)\right),
\end{aligned}
$$

where $\Theta\left(x_{0}-y_{0}\right)$ is the step function of the time difference.

Discontinuities of a two-point function may cause problems for derivatives, in particular, since they often occur simultaneously in products of two or more two-point functions. The proper procedure is, first, with the help of eq. (A.2) to represent the discontinuous parts in $F^{--}$and $F^{++}$by the continuous $F^{-+}$and $F^{+-}$times $\Theta$-functions, then to combine all discontinuities, e.g. with respect to $x_{0}-y_{0}$, into a single term proportional to $\Theta\left(x_{0}-y_{0}\right)$, and taking derivatives. One can easily check that in the two particularly relevant cases

$$
\begin{aligned}
& \frac{\partial}{\partial x_{\mu}} \int_{\mathcal{C}} \mathrm{d} z\left(F\left(x^{i}, z\right) G\left(z, x^{j}\right)+G\left(x^{i}, z\right) F\left(z, x^{j}\right)\right) \\
& {\left[\left(\frac{\partial}{\partial x_{\mu}}-\frac{\partial}{\partial y_{\mu}}\right) \int_{\mathcal{C}} \mathrm{d} z\left(F\left(x^{i}, z\right) G\left(z, y^{j}\right)-G\left(x^{i}, z\right) F\left(z, y^{j}\right)\right)\right]_{x=y}}
\end{aligned}
$$

all discontinuities exactly cancel. Thereby the values are independent of the placement of $x^{i}$ and $x^{j}$ on the contour, i.e. the values are only a function of the physical coordinate $x$.

¿From (A.2), using the Kubo-Martin-Schwinger condition [50] for two-point functions in energy-momentum representation

$$
F^{-+}(p)=F^{+-}(p) e^{-p_{0} / T}
$$

one derives the equilibrium form of two-point functions

$$
F(p)=\left(\begin{array}{cc}
F^{R}(p)-\mathrm{i} n\left(p_{0}\right) F^{\mathrm{sp}}(p) & -\mathrm{i} n\left(p_{0}\right) F^{\mathrm{sp}}(p) \\
-\mathrm{i}\left(1+n\left(p_{0}\right)\right) F^{\mathrm{sp}}(p) & -F^{A}(p)-\mathrm{i} n\left(p_{0}\right) F^{\mathrm{sp}}(p)
\end{array}\right),
$$

where $F^{\mathrm{sp}}(p)=-\mathrm{i} F^{A}(p)+\mathrm{i} F^{R}(p)=-2 \operatorname{Im} F^{R}(p)$ is the corresponding spectral function, $n\left(p_{0}\right)$ is the thermal BoseEinstein occupation number

$$
n\left(p_{0}\right)=\left[\exp \left(\left(p_{0}-e \mu\right) / T\right)-1\right]^{-1}
$$

[1] J. Schwinger, J. Math. Phys. 2 (1961) 407.

[2] G. Baym and L.P. Kadanoff, Phys. Rev. 124 (1961) 287.

[3] L.P. Kadanoff and G. Baym, Quantum Statistical Mechanics, Benjamin, 1962.

[4] L.P. Keldysh, Sov. Phys. JETP 20 (1965) 1018.

[5] E.M. Lifshiz and L.P. Pitaevskii, Physical Kinetics, Pergamon press, 1981.

[6] N.P. Landsman and Ch.G. van Weert, Phys. Rep. 145 (1987) 141.

[7] P. Danielewicz, Ann. Phys. (N.Y.) 152 (1984) 239, 305; 197 (1990) 154. 
[8] M. Tohyama, Phys. Rev. C86 (1987) 187.

[9] W. Botermans and R. Malfliet, Phys. Rep. 198 (1990) 115.

[10] A.B. Migdal, E.E. Saperstein, M.A. Troitsky and D.N. Voskresensky, Phys. Rep. 192 (1990) 179; D.N. Voskresensky, Nucl. Phys. A555 (1993) 293.

[11] J. Knoll and D.N. Voskresensky, Ann. of Phys. 249 (1996) 532.

[12] Yu.B. Ivanov, J. Knoll, and D.N. Voskresensky, Nucl. Phys. A657 (1999) 413.

[13] Yu.B. Ivanov, J. Knoll, and D.N. Voskresensky, Nucl. Phys. A672 (2000) 313.

[14] Yu.B. Ivanov, J. Knoll, H. van Hees, and D.N. Voskresensky, Phys. At. Nucl. 64 (2001) 652.

[15] J. Knoll, Yu.B. Ivanov, and D.N. Voskresensky, Ann. Phys. (N.Y.) 293 (2001) 126.

[16] Yu.B. Ivanov, J. Knoll, and D.N. Voskresensky, Phys. Atom. Nucl. 66 (2003) 1902.

[17] H. van Hees and J. Knoll, Phys. Rev. D65 (2002) 025010; D65 (2002) 105005; D66 (2002) 025028.

[18] Yu.B. Ivanov and D.N. Voskresensky, Phys. Atom. Nucl. 72 (2009) 1168; E.E. Kolomeitsev and D.N. Voskresensky, arXiv:1301.3361

[19] D.N. Voskresensky, Nucl. Phys. A849 (2011) 120.

[20] D.N. Voskresensky and A.V. Senatorov, Sov. J. Nucl. Phys. 45 (1987) 414; E.E. Kolomeitsev and D.N. Voskresensky Phys. Atom. Nucl. 74 (2011) 1316.

[21] W. Keil, Phys. Rev. D38 (1988) 152.

[22] E. Calzetta and B.L. Hu, Phys. Rev. D37 (1988) 2878.

[23] A. Hohenegger, A. Kartavtsev, and M. Lindner, Phys. Rev. D78 (2008) 085027.

[24] M. Mänson and A. Sjölander, Phys. Rev. B11 (1975) 4639.

[25] V. Korenman, Ann. of Phys. (N.Y.) 39 (1966) 72.

[26] B. Bezzerides and D.F. DuBois, Ann. Phys. (N.Y.) 70 (1972) 10.

[27] W.D. Kraeft, D. Kremp, W. Ebeling, and G. Röpke, Quantum Statistics of Charged Particle Systems , Akademie-Verlag, Berlin, 1986.

[28] J.W. Serene and D. Rainer, Phys. Rep. 101 (1983) 221.

[29] K. Chou, Z. Su, B. Hao, and L. Yu, Phys. Rep. 118 (1985) 1.

[30] J. Rammer and H. Smith, Rev. Mod. Phys. 58 (1986) 323.

[31] R. Fauser, Nucl. Phys. A606 (1996) 479.

[32] V. Spicka and P. Lipavsky, Phys. Rev. Lett. 73 (1994) 3439; Phys. Rev. B52 (1995) 14615.

[33] P. Nozièrs and E. Abrahams, Phys. Rev. B10 (1974) 4932; S. Abraham - Ibrahim, B. Caroli, C. Cardi, and B. Roulet, Phys. Rev. B18 (1978) 6702.

[34] A. Branschadel and T. Gasenzer, J. Phys. B41 (2008) 135302; M. Kronenwett and T. Gasenzer, Appl. Phys. B102 (2011) 469.

[35] G. Baym, Phys. Rev. 127 (1962) 1391.

[36] J. M. Luttinger and J. C. Ward, Phys. Rev. 118 (1960) 1417.

[37] A.A. Abrikosov, L.P. Gorkov, and I.E. Dzyaloshinski, Methods of Quantum Field Theory in Statistical Physics , Dover Pub., INC. N.Y., 1975.

[38] J.M. Cornwall, R. Jackiw, and E. Tomboulis, Phys. Rev. D10 (1974) 2428.

[39] S.T. Belyaev and G.I. Budker, Dokl. Acad. Nauk SSSR 107 (1965) 807.

[40] S.S. Adler et al., Phys. Rev. Lett. 91 (2003) 182301; J. Adams et al., Phys. Rev. Lett. 92 (2004) 052302.

[41] V.M. Galitsky, Yu.B. Ivanov, and V.A. Khangulian, Sov. J. Nucl. Phys. 30 (1979) 401.

[42] P. Danielewicz, Phys. Lett. B146 (1984) 168.

[43] P. Romatschke and U. Romatschke, Phys. Rev. Lett. 99 (2007) 172301; E. Shuryak, arXiv:0807.3033 [hep-ph].

[44] J.-S. Gagnon and J. Lesgourgues, JCAP 1109 (2011) 026.

[45] G. Torrieri and I. Mishustin, Phys. Rev. C 78 (2008) 021901.

[46] N. Anderson, Astron. Astroph. 502 (1998) 708; R. Sawyer, Phys. Rev. 39 (1989) 3804.

[47] V.V. Skokov and D.N. Voskresensky, Nucl. Phys. A828 (2009) 401; A847 (2010) 253.

[48] A. S. Khvorostukhin, V. D. Toneev, and D. N. Voskresensky, Nucl. Phys. A845 (2010) 106; Phys. Rev. C83 (2011) 035204; P. Chakraborty and J. I. Kapusta, Phys. Rev. C83 (2011) 014906; M. Bluhm, B. Kämpfer, and K. Redlich, Phys. Rev. C84 (2011) 025201.

[49] G.M. Bruun and H. Smith, Phys. Rev. A75 (2007) 043612.

[50] R. Kubo, J. Phys. Soc. Jpn. 12 (1957) 570; C. Martin and J. Schwinger, Phys. Rev. 115 (1959) 1342.

[51] S. Jeon, Phys. Rev. D52 (1995) 3591.

[52] V. S. Filinov, Y. .B. Ivanov, V. E. Fortov, M. Bonitz and P. R. Levashov, Phys. Rev. C 87 (2013) 035207.

[53] G. Aarts and J. M. Martinez Resco, Phys. Rev. D68 (2003) 085009; JHEP 0402 (2004) 061; JHEP 0503 (2005) 074.

[54] L.I. Mandelstam and M.A. Leontovich, ZhETF 7 (1937) 438.

[55] L.D. Landau and E.M. Lifshiz, Fluid Dynamics, Pergamon press, 1979.

[56] F. Karsch, D. Kharzeev, and K. Tuchin, Phys. Lett. B663 (2008) 217.

[57] C. Itzykson and J.-B. Zuber, Quantum Field Theory, McGraw-Hill, 1980. L.D. Landau and E.M. Lifshiz, Statistical Physics, Part 1, Pergamon press, 1980.

[58] Yu. B. Rumer and M.Sh. Rivkin, "Thermodynamics, Statistical Physics and Kinetics", Nauka, Moscow, 1977, in Rus.

[59] P. Romatschke, Int. J. Mod. Phys. E 19, 1 (2010) arXiv:0902.3663 [hep-ph]]. 